\title{
En bloc Pancreaticodudenectomy with Colectomy for Locally Advanced Right Sided Colon Cancer
}

\author{
Ramesh Singh Bhandari, ${ }^{1}$ Paleswan Joshi Lakhey, ${ }^{1}$ Parsu Ram Mishra ${ }^{1}$ \\ 'Surgical Gastroenterology Units, Department of Surgery, Tribhuvan University Teaching Hospital, Kathmandu, Nepal.
}

\begin{abstract}
A 57 year old male presented to our outpatient clinic with history of on and off melena, weight loss and decreasing appetite for 10 months duration and a noticeable mass on the right upper quadrant. Abdominal examination revealed an intra-abdominal lump in right upper quadrant which was subsequently evaluated by colonoscopy, which revealed an ulcero-proliferative growth in the hepatic flexure and the biopsy from it confirmed well-differentiated adenocarcinoma. Contrast enhanced computed tomography demonstrated hepatic flexure mass with possible invasion into adjacent duodenum without features of advanced disease. After completion of necessary preoperative assessment and investigations, patient was explored with curative intent and underwent extended right hemicolectomy with en bloc pancreaticodudenectomy. Patient was discharged on $10^{\text {th }}$ postoperative day and at 14 months follows up; he was doing well without any evidence of recurrence.
\end{abstract}

Keywords: colectomy; locally advanced; pancreaticoduodenectomy.

\section{INTRODUCTION}

Extracolonic involvement of adjacent organ is a known phenomenon for colorectal cancers. However extracolonic tumor spread by right colon cancer is not a frequently seen condition. ${ }^{1,2}$ Right colon cancer can involve duodenum and pancreas and this condition represents a dilemma for treating surgeon as the optimal procedure for this condition has not been defined very well yet. ${ }^{2}$ However, many reports of right hemicolectomy with en bloc pancreaticodudenectomy has been published and has shown good perioperative results with long term survival. ${ }^{3}$ Here, we report a similar case of right colon cancer invading into the duodenum and was managed successfully with right hemicolectomy and en bloc pancreaticodudenectomy.

\section{CASE REPORT}

A 57 year old male presented to our outpatient clinic with history of on and off melena, weight loss and decreasing appetite for 10 months duration and noticeable mass on the right upper quadrant which in fact was gradually increasing on size, on his own words.
He didn't have any major surgical or medical morbidity. On examination, he was thin built but otherwise no other abnormal finding on general physical examination was detected. Abdominal examination did reveal an intra-abdominal lump in right upper quadrant but very doubtful mobility and poorly defined margins. On this basis, patient was subjected to colonoscopy, which revealed an ulcero-proliferative growth in the hepatic flexure and the biopsy from which subsequently revealed well-differentiated adenocarcinoma. In the meantime, contrast enhanced computed tomography (CT) scan was performed on him, which confirmed a hepatic flexure mass with suspicious invasion into duodenum (Figure 1). However, no significant lymphadenopathy and any evidence of metastatic disease were noted. Following completion of necessary preoperative assessment and investigations, patient was explored with curative intent. Intraoperatively, there was a hepatic flexure mass grossly invading into adjacent duodenal wall without

Correspondence: Dr. Ramesh Singh Bhandari, Associate Professor, Department of Surgery, Tribhuvan University Teaching Hospital, Kathmandu, Nepal. Email: rsbhandari09@gmail.com, Phone : +977-9841270203. 
gross lymphadenopathy and metastatic disease, which would stop us proceeding to radical surgery (Figure 2). As the disease was locally advanced but resectable we proceeded ahead with extended right hemicolectomy with en bloc pancreaticodudenectomy (Figure 3).
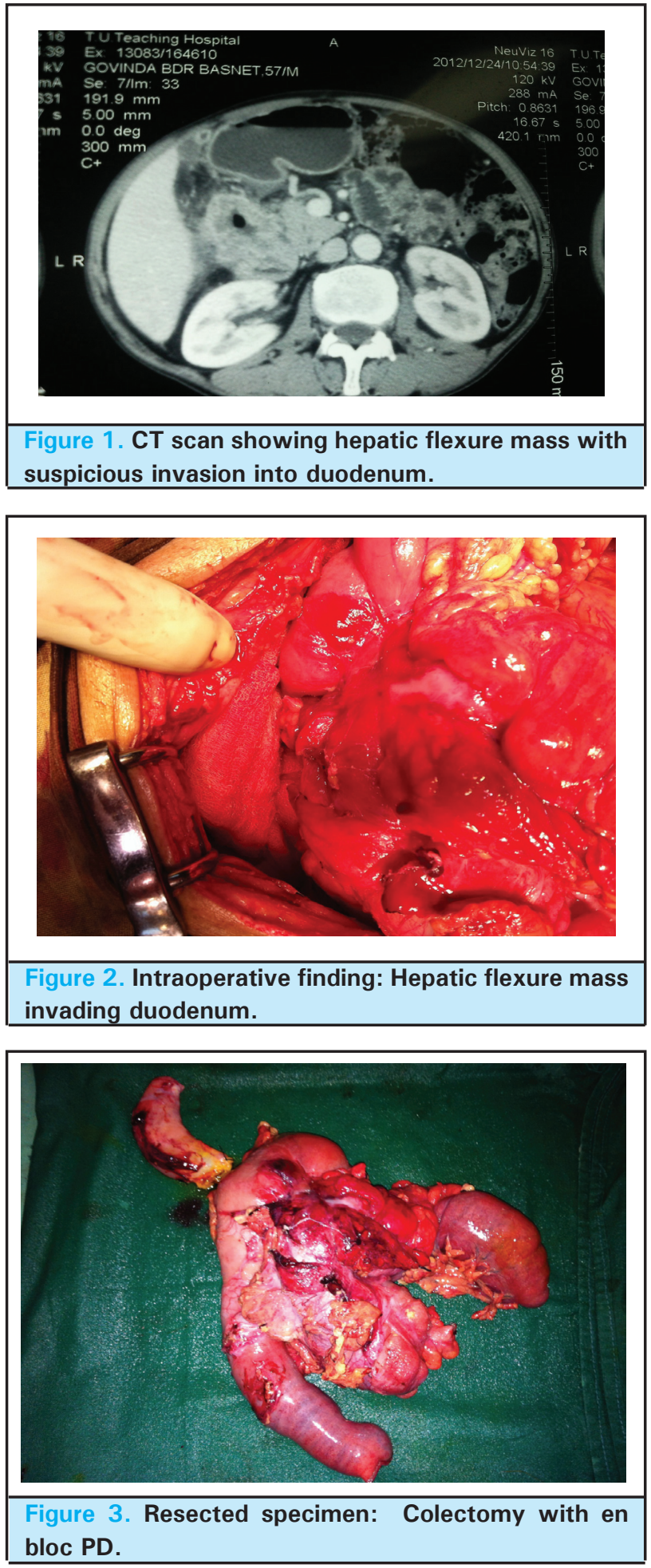

Postoperative period remained uneventful and patient was discharged on $10^{\text {th }}$ postoperative day. Final histopathology report confirmed poorly differentiated adenocarcinoma with duodenal invasion, free of margins, 0 out of 28 dissected nodes positive (T4bNO disease). On follow up at 14 months, patient was doing well with out any evidence of recurrence on basis of normal CEA and imaging.

\section{DISCUSSION}

Colorectal cancer invading adjacent organs is not an infrequent situation seen in around 5.5- $16.7 \%$ of all colorectal cancers. ${ }^{4}$ However, invasion of right colon cancer into duodenum and or pancreas is not a frequent condition and it can present as a diagnostic dilemma. Right colon cancer invading into these adjacent structures is considered as T4 stage disease. ${ }^{5}$ Locally advanced cancers invading to adjacent organs are frequently considered incurable. Colorectal cancers are exceptions to this rule as locally advanced colorectal cancers can often be completely resectable. ${ }^{6}$ Croner et al showed that clinical suspicion of the local invasion by colon cancer into adjacent organs when microscopically examined revealed neoplastic infiltration in $53.4 \%$ cases and inflammatory adherence in $46.6 \%$ of cases. ${ }^{7}$ Berrospi et al also showed $50 \%$ of adherence of locally advanced colon cancer as malignant infiltration. ${ }^{8}$ Diagnosis of locally advanced colon tumors in the past usually occurred intraoperatively. However, availability of the advanced imaging techniques has helped to detect them preoperatively except for small number of cases when they present in emergencies with acute problems like bleeding and obstructions. Abdominal CT Scan can reveal local invasion as high as in $86 \%$ of cases. $^{9}$ Role of imaging is not just diagnostic of invasion into pancreas and or duodenum but to guide for selection of patients in whom total cytoreduction surgery is feasible.

Locally advanced right sided colon cancer invading the duodenum and or pancreas requires an en bloc pancreaticodudenectomy and the complexity associated with this surgery seems to have discouraged surgeons to perform such a procedure. ${ }^{8}$ However PD has become much safer nowadays. High volume centers display mortality rates after PD of $1-6 \% .{ }^{10-12}$ Similarly, in last decades there has been large number of reports in the form of case reports and series on PD with locally advanced colon cancer showing a good perioperative outcome and equally a good long-term survival. ${ }^{13}$

Koe et al also suggested right hemicolectomy with en bloc PD for large tumors invading the duodenum or if there is any suspicion of malignant infiltration. ${ }^{14}$ Curley et al reported four of seven patients having en bloc PD living free of recurrence at a median follow up of 42 months. ${ }^{15}$ Saiura et al described 12 patients of colon 
cancer invading the pancreatic head and undergoing en bloc PD with colon resection. ${ }^{16}$ Of them, five patients survived more than ten years without local recurrence. Some reports of partial duodenal resection for locally advanced colon cancer has been published but it has been found to be associated with poor outcome when compared with PD. ${ }^{17}$ This might suggest the need of en bloc resection of the adjacent organs including the lymphatic drainage. However, some authors still do recommend partial duodenal resection when duodenum only is involved without involvement of the ampulla of vater in very high-risk patients. ${ }^{18}$ Cirocchi et al published a review on right hemicolectomy with PD vs. local duodenal resection for this group of patients. ${ }^{17}$ Out of 53 patients of locally advanced right colon cancer patients undergoing $\mathrm{RO}$ resections, 39 had colectomy with en bloc PD, 10 had partial duodenal resection with ileal flap closure and 4 with direct suture repair after partial duodenal resection. The 30-day postoperative morbidity was similar in both the groups; however 5 year overall survival was $50 \%$ for en bloc PD, $25 \%$ for partial duodenal resection with ileal flap closure and $0 \%$ for primary suture closure of the duodenum. This finding strongly supported the concept of doing en bloc PD with colon resection for locally advanced colon cancer.

\section{CONCLUSION}

Locally advanced right colon cancer invading into duodenum and or pancreas not necessarily means incurable disease. When indicated and taken apart the comorbidities, this procedure in very high volume centers can safely be performed with excellent outcomes and giving patients a chance of long term survival. If a surgeon is not familiar with this procedure, it is recommended to close the abdominal wall and refer patient to specialized center as the surgeon with limited experience might judge tumor unresectable and perform bypass reducing the chance of cure.

\section{REFERENCES}

1. Heslov SF, Frost DB. Extended resection for primary colorectal carcinoma involving adjacent organs or structures. Cancer1988;62:1637-40.

2. Lianwen $Y$, Jianping Z, Guoshun S, Dongcai L, Jiapeng Z. Surgical treatment for right colon cancer directly invading the duodenum. The American surgeon 2009;75:385-88.

3. Song XM, Wang L, Zhan WH, et al. Right Hemicolectomy combined with pancreaticodudenectomy for the treatment of colon carcinoma invading the duodenum or pancreas. Chin Med J 2006;119:194-7.

4. Staniunas RJ, Schoetz: Extended resection for carcinoma of the colon and rectum. Surg Clin North Am 1993;73:117-129.

5. Colon and Rectal cancer staging. American Joint Committee on Colon Cancer. $7^{\text {th }}$ edition.

6. Lopez MJ, Monafo WW. Role of extended resection in the initial treatment of locally advanced colorectal carcinoma. Surgery 1993;113:365- 72.

7. Croner R, Merkel S, Papadopoulos T, Schellerer V, Hohenberger W, Goehl J. Multivisceral resection for colon carcinoma. Dis Colon Rectum 2009;52:1381-6.

8. Berrospi F, Celis J, Ruiz E, Payet E. En bloc pancreaticoduodenectomy for right colon cancer invading adjacent organs. J Surg Oncol 2002;79:194-8.

9. Grossmann I, Klaase JM, Avenarius JK, de Hingh IH, Mastboom WJ, Wiggers T. The strengths and limitations of routine staging before treatment with abdominal CT in colorectal cancer. BMC Cancer 2011;11:433.

10. Edge SB, Schmieg RE Jr, Rosenlof LK, Wilhelm MC. Pancreas cancer resection outcome in American university centres in 1989- 1990. Cancer 1993:71:3502-8.
11. Cameron JL, Riall TS, Coleman J, Belcher KA. One thousand consecutive pancreaticodudenctomies. Ann Surg 2006;244:10-5

12. Gordon TA, Burleyson GP, Tielsch JM, Cameron JL. The effects of regionalization on cost and outcome for one general high risk surgical procedure. Ann Surg 1995;221:43-9.

13. Landmann RG, Weiser MR. Surgical management of locally advanced and locally recurrent colon cancer. Clin Colon Rectal Surg 2005;18:182-9.

14. Koea JB, Conlon K, Paty PB, Guillem JG, Cohen AM. Pancreatic or duodenal resection or both for advanced carcinoma of the right colon: is it justified? Dis Colon Rectum 2000;43:460-5.

15. Curley SA, Evans DB, Ames FC: Resection for cure of carcinoma of the colon directly invading the duodenum of pancreatic head. J Am Coll Surg 1994;179:587-592.

16. Saiura A, Yamamoto J, Ueno M, Koga R, Seki M, Kokudo N. Long term survival in patients with colon cancer after en bloc pancreaticodudenectomy and colectomy. Dis Colon Rectum 2008;51:460-5.

17. Cirocchi R, Partelli S, Castellani E, Renzi C, Parisi A, Noya G, Falconi M. Right hemicolectomy plus pancreaticoduodenectomy vs partial duodenectomy in treatment of locally advanced right colon cancer invading pancreas and/or only duodenum. Surgical Oncology 2014;23:92-98.

18. Lianwen Y, Jianping Z, Guoshun S, Dongcai L, Jiapeng Z. Pedicled Ileal flap for duodenal defect after right hemicolectomy. Hepatogastroenterology 2010;57:493-6. 